DOI: 10.18027/2224-5057-2017-7-3s1-22-25

Цитирование: Панкратов А. Е., Деньгина Н. В. Облучение лимфатических коллекторов при немелкоклеточном раке легкого: широкое, избирательное или никакого? // Злокачественные опухоли. - 2017. - Т. 7. - № 3, спецвыпуск 1. - С. 22-25.

\title{
Облучение лимфатических коллекторов при немелкоклеточном раке легкого: широкое, избирательное или никакого?
}

\author{
А.Е. Панкратов, Н.В. Деньгина
}

Информация об авторах:

\author{
Александр Е. Панкратов, ООО «ПЭТ-Технолоджи Балашиха», Центр онкологии и лучевой терапии, г. Балашиха, \\ e-mail: pediatrician@yandex.ru
}

Наталья В. Деньгина, гБУЗ Ульяновский областной онкологический диспансер, г. Ульяновск, e-mail: indigo171201@yahoo.com

\begin{abstract}
Резюме: В настоящее время существует две основных парадигмы в выборе объема облучения лимфоколлектора при немелкоклеточном раке легкого: 1. Включение в объем облучения только очевидно пораженных тканей легкого и регионарных лимфоузлов, вовлеченных в опухолевый процесс с исключением из полей облучения лимфоузлов с гипотетическим микрораспространением опухолевых клеток. Основная цель в таком выборе - возможность подведения максимальных курабельных доз излучения (более 60 Гр) за счет уменьшения общего объема излучения и снижения лучевой токсичности. 2. Включение в объем облучения как очевидно пораженных лимфоузлов, так и тех, которые теоретически могут иметь очаги микрометастазирования. Основная логика такого выбора заключается в том, что области, требующие подведения высоких доз (более 60 Гр), достаточно малы, а очаги микрометастазирования поддаются дозам облучения не более 50 Гр и, таким образом, может быть увеличена выживаемость. Вопрос, какой же логике следовать при выборе объема облучения лимфоколлекторов, и посвящена данная работа.
\end{abstract}

Ключевые слова: немелкоклеточный рак легкого, лимфоколлектор, лучевая терапия

Рак легкого в онкологической заболеваемости населения занимает второе или третье место в зависимости от региона и пола пациента и первое место в смертности от онкологических заболеваний. При этом на немелкоклеточный рак легкого (НМКРЛ) приходится более $80 \%$ от всех типов рака легкого. На протяжении десятилетий совершенствуются методы лечения данной патологии. И, если в лекарственном лечении основным направлением является создание новых препаратов, которые уже используются с учетом молекулярно-генетических особенностей опухоли, то в области лучевой терапии главным направлением является создание новых технологий подведения лечебного пучка к пораженным тканям при минимальном воздействии на непораженные. Учитывая относительную радиорезистентность опухолевой ткани немелкоклеточного рака легкого, необходимо подведение достаточно высоких доз облучения для получения лечебного эффекта, но вместе с тем высокие дозы облучения и специфика локализации процесса требуют предельной четкости в понимании вы- бора объема лучевого воздействия. Это важно, т. к. высокая лучевая токсичность в отношении таких критических структур, как миокард и неповрежденная паренхима легких, может быть причиной худшего прогноза у пациентов, которым выполнена лучевая терапия в объеме, большем, чем достаточно в сравнении с пациентами, которым выполнено облучение только областей с высоким риском прогрессии или рецидива злокачественного процесса. Таким образом, основная цель при определении объема лучевой терапии при НМКРЛ - не только выяснить, что облучать, а также что можно и лучше не облучать. Современные руководства, такие как NCCN и ESMO, которым следуют большинство онкологов, однозначно исключают применение лучевой терапии на ранних стадиях резектабельного рака легкого при N0 и N1 - стадии поражении лимфоколлекторов при радикально выполненной операции. Обоснованием этому решению послужили ПОРТ-метаанализы 1998 и 2005 гг., которые показали, что применение послеоперационной лучевой терапии при ранних стадиях 
НМКРЛ ухудшает общую выживаемость пациентов [1, 2]. Однако последующая количественная оценка метаанализа ПОРТ показала неполноценность методов в примененном метаанализе и результатом исследования оказалось то, что выводы метаанализа не имеют отношения к современным пациентам [3]. При локально распространенных стадиях речь идет о сочетании хирургии, химиотерапии и лучевой терапии. При этом наибольшую сложность представляет вопрос о воздействии не на первичный очаг, а на регионарные лимфоузлы. Где находится та граница, на которой следует остановиться при выборе уровня облучаемых лимфоузлов? В этом вопросе мы и попробуем разобраться. Во-первых определимся, что лучевая терапия может выполняться как в послеоперационном периоде послеоперационная радиотерапия (ПОРТ), так и с паллиативной целью. Во-вторых, остановим наше внимание только на объеме воздействия на лимфоколлекторы в зависимости от степени распространенности процесса. Для начала определимся, в каких ситуациях мы вовсе не будем применять лучевую терапию в послеоперационном периоде. На этот вопрос есть ответ в рандомизированном исследовании ANITA 2008 г., показавшем, что ПОРТ целесообразна только при III N2 - стадии и в сочетании с химиотерапией [4]. При пересмотре базы данных национального канцер-регистра США и оценки почти 113 тыс. пациентов с НМКРЛ I-III ст., которым выполнено хирургическое лечение с резекцией R1-R2, выявлено, что послеоперационная ХЛТ дает лучшие результаты при III стадии [5]. С целью оценки лучевой токсичности ПОРТ на сердце был выполнен мультивариантный анализ, который показал, что использование ПОРТ было связано с увеличением смертности от болезней сердца. При этом ассоциация была подтверждена в когорте, которая диагностировалась с 1983 по 1988 год, но не для когорты, которая диагностировалась с 1989 по 1993 год. Авторы анализа отмечают, что это может быть связано с улучшением лечебного планирования и качеством подведения излучения к органам грудной клетки [6]. Метаанализ ПОРТ 2013 показал эффективность ПОРТ при III N2 стадии, причем результаты были существенно лучше при выполнении облучения на линейных ускорителях [7]. Таким образом, выполнение облучения лимфоколлектора при N2-стадии целесообразно, независимо от объема хирургического лечения, и предпочтительно его выполнение на линейных ускорителях как минимум с 3D-планированием. Теперь, собственно, об объеме облучения лимфоколлекторов, что, в общем, и является главным вопросом. Стандартно многие годы в плане послеоперационной лучевой терапии было облучение всего средостения, надключичной области со стороны поражения и культи бронха со стороны поражения, независимо от их пораженности процессом [8]. В исследовании, выполненном в Memorial Sloan-Kettering Cancer Center, были оценены пациенты с НМКРЛ, которым кроме первичного очага выполнено избирательное облучение только пораженных лимфоузлов. Двухлетний контроль над опухолевым ростом в лимфоузлах составил 85-91\% [9]. Данное исследование позволяет авторам рекомендовать ограничиваться облучением только пораженных лимфоузлов. Какие же лимфоузлы считать пораженными для определения воздействия на них? Безусловно те, в которых процесс верифицирован цитологическим или гистологическим исследованием. Кроме того, рентгенологическим признаком поражения лимфоузла считается увеличение его линейных размеров до 15 мм и более. Однако процесс может быть уже и в лимфоузлах нормальных размеров, а в отдельных увеличенных лимфоузлах может не оказаться опухолевых клеток. Наиболее ценным методом выявления опухолевой инвазии в лимфоузлы можно считать ПЭТ-КТ с 18-ФДГ. Многоцентровое исследование из Нидерландов, авторы которого выполняли облучение лимфоколлектора только с выявленным по данным ПЭТ-КТ поражением, зарегистрировали всего $3 \%$ рецидива в лимфоузлах и низкую частоту лучевого эзофагита (12\%) по сравнению с таковыми при использовании только КТ-данных, когда частота рецидивов в лимфоузлах достигала $11 \%$ [10]. Итак, на основании приведенных исследований можно сказать, что в область облучения должны войти лимфоколлекторы с пораженными лимфоузлами. При этом речь будет идти о заболевании стадии N2. В ответе на вопрос, какой уровень лимфоузлов будет соответствовать локализации в той или иной доле легкого первичного очага, нам поможет атлас лимфоузлов для определения объема CTV (clinical target volume, клинический объем облучения) [11], основанный на классификации лимфатических узлов при стадировании рака легкого Mountain и Dresler 12]. Насколько поле облучения будет большим или ограниченным, будет зависеть от объема поражения и отчасти от гистологического подтипа НМКРЛ. Так, микроскопическое распространение аденокарциномы составляет 2,69 мм и плоскоклеточного рака - 1,48мм [13]. Соответственно, увеличение края от GTV (gross tumor volume, основной объем опухоли) до CTV рекомендуется прибавлением 8 мм для аденокарциномы и 6 мм для плоскоклеточного рака, что обеспечит покрытие 95\% объема с учетом микроскопического распространения [14]. Для обеспечения 95\% покрытия пораженных лимфоузлов при КТ планировании на изначальный контур лимфоузлов необходимо сделать прибавку к краю 5 мм [15]. При КТ-планировании лечения визуализация опухоли будет зависеть от выбранного «окна просмотра». Так, для лучшей визуализации и стандартизации процесса рекомендуется использовать следующие установки: $\mathrm{W}=1600, \mathrm{~L}=-600$ для легочной паренхимы и $\mathrm{W}=400, \mathrm{~L}=20$ для средостения [16]. А теперь можно вернуться к рекомендациям ICRU-50 и ICRU-60 международной комиссии по радиационным единицам и измерениям. Для облегчения создания 3D-планирования было предложено определить объемы мишеней, подлежащих лучевому воздйствию: 1) GTV - основной объем опухоли, который 
состоит из макроскопической опухоли, визуализируемой в т.ч. доступными средствами получения медицинских изображений; 2) CTV - клинический объем мишени, представляющий GTV с расширением с учетом субклинического распространения опухоли; 3) PTV - планируемый объем мишени, который представляет собой расширение CTV с учетом внутреннего движения мишени, неточностей укладки и других неопределенностей. Это окончательный объем облучения. PTV - это геометрическая структура, отвечающая за то, что 95\% уровень ее изодозы должен привести к адекватному распределению дозы в CTV; 4) ITV - внутренний объем мишени (концепция, введенная в ICRU-62, чтобы включить движение опухоли как отдельную сущность в определении мишени) $[17,18]$. Соответственно этим рекомендациям и строится план лучевой терапии; и то, что мы получили в финале анализа литературы, соответствует рекомендациям ICRU, с которых, возможно следовало начать.

\section{Выводы}

1. Послеоперационная лучевая терапия необходима только при N2-стадии процесса либо при неполной резекции (R1/R2).
2. Применение лучевой терапии при ранних стадиях ухудшает прогноз.

3. С целью уточнения опухолевой инвазии в лимфоузлах и оценки распространенности целесообразно использовать все доступные методы диагностики: биопсия лимфоузлов, ПЭТ-КТ.

4. В область облучения должны быть включены только пораженные лимфоузлы с учетом уровня поражения.

5. Надключичные лимфоузлы также подлежат облучению при их поражении.

6. Лучевая терапия должна сочетаться с химиотерапией.

7. Дополнительная прибавка от GTV до CTV на область первичного очага составляет 8 мм при аденокарциноме и 6 мм при плоскоклеточном раке, на область пораженных лимфоузлов - 5 мм.

8. При создании РTV необходимо учитывать смещаемость облучаемых объемов вследствие дыхания.

9. Выполнять лучевую терапию органов грудной клетки с меньшими рисками лучевых осложнений и более высокой эффективностью важно с применением современной техники (линейные ускорители) и адекватных систем планирования (минимум 3D).

10. Финальный план лучевого лечения должен соответствовать рекомендациям ICRU, если нет противоречий.

\section{Литература • References}

1. PORT Meta-analysis Trialists Group. Postoperative radiotherapy in non-small-cell lung cancer: Systematic review and meta-analysis of individual patient data from nine randomised controlled trials. Lancet. 1998; 352: 257-63.

2. PORT Meta-analysis Trialists Group. Postoperative radiotherapy for non-small-cell lung cancer. Lang. Cancer. 2005; 47: 81-3.

3. Abuodeh YA, Naghavi A. O., DeMarco M, Tonner B, Feygelman V, Stevens CW, Perez BA, Dilling TJ Quantitatively Excessive Normal Tissue Toxicity and Poor Target Coverage in the PORT Meta-Analysis (MA): A Dosimetric Study. Int JRadiat Oncol Biol Phys 2016;96:442.

4. Douillard JY, Rosell R, De Lena M, et al. Impact of postoperative radiation therapy on survival in patients with complete resection and stage I, II, or IIIA non-small-cell lung cancer treated with adjuvant chemotherapy: the adjuvant Navelbine International Trialist Association (ANITA) Randomized Trial. Int JRadiat Oncol Biol Phys 2008;72:695-701.

5. Osarogiagbon, R. U., Lin, C. C., Smeltzer, M. P. et al, Prevalence, prognostic implications, and survival modulators of incompletely resected non-small cell lung cancer in the U.S. National Cancer Data Base. J Thorac Oncol. 2016;11: e5 - e16.

6. Lally B. E., Detterbeck F. C., Geiger A. M., Thomas C. R. Jr, Machtay M., Miller A. A. et al. The risk of death from heart disease in patients with non-small cell lung cancer whoreceive postoperative radiotherapy: analysis of the Surveillance, Epidemiology, and End Results database. Cancer. 2007; 110 (4): 911-7.

7. Billiet C., Decaluwй H., Peeters S., Vansteenkiste J., Dooms C., Haustermans K. et al. Modern postoperative radiotherapy for stage III non-small cell lung cancer may improve local control and survival: A meta-analysis. Radiother. Oncol. 2013; 110 (1): 3-8.

8. Emami B, Graham MV. Lung. In: Perez C, Brady LW, editors. Principles and practice of radiation oncology. New York: LippincottRaven; 1998. p. 1181-1220.

9. Rosenzweig $\mathrm{K}$ et al. Elective nodal irradiation in the treatment of non-small-cell lung cancer with three-dimensional conformal radiation therapy. Int J Radiat Oncol Biol Phys. 2001 Jul 1;50 (3):681-5.

10. Van LJ, De Ruysscher D, Wanders R, Boersma L, Simons J, Oellers M, Dingemans AM, Hochstenbag M, Bootsma G, Geraedts W, Pitz C, Teule J, Rhami A, Thimister W, Snoep G, Dehing-Oberije C, Lambin P: Selective nodal irradiation on basis of (18) FDG-PET scans in limited-disease small-cell lung cancer: a prospective study. Int J Radiat Oncol Biol Phys 2010, 77: 329-336.

11. Martinez-Monge R, Fernandes PS, Gupta N, Gahbauer R: Cross-sectional nodal atlas: a tool for the definition of clinical target volumes in three-dimensional radiation therapy planning. Radiology 1999, 211: 815-828. 
12. Mountain CF, Dresler CM. Regional lymph node classification for lung cancer staging. Chest 1997;111:1718-1723.

13. Giraud $P$, Antoine M, Larrouy A: Evaluation of microscopic tumor extension in non-small-cell lung cancer for three-dimensional conformal radiotherapy planning. Int J Radiat Oncol Biol Phys 48:1015-1024, 2000.

14. Senan S, Chapet O, Lagerwaard FJ, Ten Haken RK: Defining target volumes for non-small cell lung carcinoma. Semin Radiat Oncol 2004, 14: 308-314. 10.1016/j. semradonc. 2004.07.004.

15. van Sornsen de Koste JR, Lagerwaard FJ, Nijssen-Visser MRJ: Which margins are necessary for incorporating mediastinal nodal mobility in involved field radiotherapy for lung cancer? Int J Radiat Oncol Biol Phys 53:115-119, 2002.

16. Giraud P: Influence of CT images visualization parameters for target volume delineation in lung cancer (abstr). Radiother Oncol 56:39, 2000 (suppl 1).

17. International Commission on Radiation Units and Measurements: Prescribing, Recording, and reporting photon beam therapy. Bethesda, MD: ICRU Report 50, 1993.

18. International Commission of Radiation Units, Measurements, report no. 62: Prescribing, recording and reporting photon beam therapy (supplement to ICRU Report 50). Bethesda, MD: ICRU, 1999. 